\title{
L'Opéra au service de la Nation. La relecture rhétorique de la Guerre de l'Opéra de Jacques Cazotte
}

\author{
Abstract \\ The Opera in the Service of the Nation. The Rhetorical Lecture of Guerre de l'Opéra \\ by Jacques Cazotte
}

The premiere of Serva Padrona by Pergolese at the Paris Opera divided deeply the French society of the Age of Enlightenment. The famous Querelle des Bouffons has become the nucleus of a lively debate, from which, what is to be emphasized, none of the sides was triumphant. In his treatise Guerre de l'Opéra, Jacques Cazotte, emphasizing his impartiality, stands in defense of French music.

By proving the superiority of Mondonville's works, Cazotte is aware that only strong arguments can convince the philosophers. Accused by many of being "scientific" and in consequence, being incapable of expressing all the nuances of human passions, the French music, thanks to the ability to perform rhetorical functions, reserved, as it may seemingly appear, for forewords and literature, can not only conform to the rules, but also can please and touch. The French Opera, as Cazotte poves, fills perfectly the rhetorical target of persuasio with an intelligent and effective rhetoric and expresses the spirit and sensitivity of France.

The purpose of this article is to reflect on how Cazotte defends French music from the fashion for Italian music, which had a strong influence on the French aesthetics of the Age of Enlightenment, as well as from the philosophers of the epoch who would discredit at all costs the work of French composers in the eyes of the audience.

Keywords: Jacques Cazotte, French Opera, French Enlightenment, rhetoric

La guerre commence d'une façon anodine. Le $1^{\text {er }}$ août 1752, une troupe italienne, les célèbres « bouffons », viennent jouer à l'Académie royale de musique à Paris La serva padrona, un intermezzo en deux parties de Giovanni-Battista Pergolesi, sur un livret de Gennaro Antonio Federico. Le spectacle déclenche une vive discussion qui oppose les partisans de la musique française à ceux de la musique italienne. Selon les enthousiastes des Italiens, l'originalité de leurs 
méthodes fait apparaitre quelque peu démodée la musique française traditionnelle alors incarnée par Lulli et Rameau. Dans les propos des fervents admirateurs du style musical français, le spectacle italien revêt la forme d'un attentat violent non seulement au goût musical des Français, mais aussi à toute l'esthétique et l'identité françaises. Dans les années 1752-1754, dans la presse parisienne, s'instaure la fameuse "Querelle des Bouffons 》, où les Encyclopédistes, les hommes des Lumières, se retrouvent du côté des Italiens, le plus décidé et le plus talentueux des adversaires de l'opéra français étant Jean-Jacques Rousseau qui, avec sa Lettre sur la musique française, publiée en 1753, provoque un vrai orage. Dans son texte, Rousseau accuse la musique française d'être dépourvue de mélodie et met en valeur la supériorité de la musique italienne, qui, grâce à « la douceur de la langue », « la hardiesse des modulations » et « l'extrême précision de la mesure » transmet à l'âme tous les sentiments qu'elle veut y éveiller'. Par la multiplication des ouvrages qui y sont engagés, par la force des arguments de nature musicale, rhétorique et... nationale, cette querelle dépasse très vite le domaine musical pour initier un conflit acharné entre la tradition et le progrès et entre la nation française et la nation italienne. Cette vive discussion se transforme en une vraie guerre argumentative. C'est en tout cas ainsi que la considère Jacques Cazotte dans son traité La Guerre de l'opéra, une brochure publiée en février 1753 où Cazotte, en défendant la musique française, met en évidence que la tradition musicale des Français est complétement différente de celle des Italiens parce qu'elle découle d'une sensibilité nationale spécifique. Certes, ce discours s'inscrit aussi dans la polémique privée entre Cazotte et Jean-Jacques Rousseau, qui, quant à lui, va affirmer que « les François n'ont point de Musique et n'en peuvent avoir ; ou que si jamais ils en ont une, ce sera tant pis pour eux $»^{2}$, mais, avant tout, il participe à la guerre dont l'intensité et la ferveur attirent l'attention de Cazotte :

Le feu est dans tous les Coins de l'Opéra. La Musique Italienne y est aux prises avec la Musique Françoise. Imaginez tous les desordres d'une guerre en même tems étrangere \& civile. Des intrigues, des brigues, des factions, des cabales, des hauts, des bas, des révolutions étonnantes; la fortune déclarée d'abord pour l'étranger sans se donner le tems de choisir, chancelante ensuite entre les deux partis, pour se livrer bien-tôt à nous, sans trop sçavoir pourquoi. Des joies outrées, des yvresses, des triomphes passagers, des chûtes inopinées, des projets fols, des desespoirs extravagans. Voilà le foible crayon de ce qui vient de se passer sous nos yeux au Théatre lyrique ${ }^{3}$.

Se servir de la musique à des fins politiques n'est pas une chose nouvelle. La fondation de l'Académie royale de musique avait, évidemment, une grande importance culturelle, mais elle s'expliquait aussi par une rivalité entre la France et l'Italie dans le domaine de la création musicale et, par conséquent, de la supériorité civilisatrice. Dans le privilège accordé par Louis XIV à Pierre Perrin, en juin 1669, établissant l'Académie royale de Musique à Paris, la musique italienne

1 J.-J. Rousseau, Lettre sur la musique française [dans :] Euvres complètes, t. V, Paris 1995, p. 303-305.

2 Ibid., p. 328.

3 J. Cazotte, La Guerre de l'opéra. Lettre écrite à une dame de province, [sans lieu] 1753. Pour toutes les citations du XVIII ${ }^{\mathrm{e}}$ siècle, la graphie originale est respectée. 
est désignée comme le facteur générateur de l'idée de fonder en France une institution semblable aux diverses institutions musicales qui existent en Italie. Dans le document signé de sa main, le roi déclare : « Nous avons très-humblement fait remonstrer, que depuis quelques années les Italiens ont establi diverses Académies dans lesquelles il se fait des Représentations en musique qu'on nomme Opéra $»^{4}$. L'objectif principal de la décision royale est de contribuer à l'avancement des arts en France, mais aussi de mettre en valeur le rôle et l'influence de la musique française sur la culture européenne et souligner que ni l'Italie, ni aucun autre pays, n'a le monopole en matière de spectacles d'opéra.

Pourtant, lorsqu'un siècle plus tard, sur la vague d'enthousiasme suscité par les spectacles en musique montés en France par des Italiens, il peut paraître que l'esthétique musicale italienne prime sur le style français, Cazotte décide de prendre la parole dans cette discussion acharnée. À cette époque, il n'est pas étonnant de voir un écrivain ou un philosophe participer au débat au sujet de la musique.

\section{La méthode rhétorique de l'analyse de la musique}

Depuis les années vingt du XVIII ${ }^{\mathrm{e}}$ siècle, on postule la capacité d'un large public de juger de la valeur d'une œuvre d'art. Le critère qu'on applique à l'évaluation de l'art est purement rhétorique. L'abbé Dubos précise que l'unique but de l'art est de plaire et d'émouvoir, deux fonctions de base de la rhétorique. Ainsi, Dubos, sans offenser les spécialistes en critique littéraire et artistique, s〉oppose aux dogmes de la composition des œuvres et de leur réception ${ }^{5}$. Un des signes distinctifs de l'époque des Lumières est le renoncement au jugement des œuvres d'art au moyen de règles connues par les gens de métier ${ }^{6}$.

Par conséquent, dans les écrits de Cazotte sur la musique, il faut remarquer une nuance argumentative. Les thèses qu'il cherche à défendre sont présentées à l'aide de la terminologie rhétorique. Ceci ne devrait pas étonner parce qu'un autre trait caractéristique de la pensée des Lumières, est son profond enracinement dans l'ancien art oratoire. L'utilité universelle de la rhétorique dans tout discours critique sur les beaux-arts est soulignée par l'Encyclopédie qui explique : « La rhétorique est d'un usage fort étendu, elle renferme tout ce qu'on appelle en

${ }^{4}$ Ch. Nuitter, E. Thoinan, Les Origines de l'Opéra français, Paris 1886, p. 97.

5 «Veritablement ceux qui ne sçavent point l'art, ne sont pas capables de remonter jusques aux causes qui rendent un mauvais poëme ennuyeux. Ils ne sçauroient en indiquer les fautes en particulier. Aussi ne prétends-je pas que l'ignorant puisse dire precisement en quoi le Peintre ou le Poëte ont manqué, \& moins encore leur donner des avis sur la correction de chaque faute, mais cela n'empêche pas que l'ignorant ne puisse juger par l'impression que faait sur lui un ouvrage composé pour lui plaire \& pour l'interesser, si l'Auteur a réussi dans son entreprise $\&$ jusqu'à quel point il y a réussi », L'Abbé Dubos, Réflexions critiques sur la poésie et la peinture, seconde partie, Paris 1719, p. 343.

${ }^{6}$ A. Lontrade, Le plaisir esthétique. Naissance d'une notion, Paris 2004, p. 58. 
françois belles-lettres $»^{7}$. Au XVIII ${ }^{\mathrm{e}}$ siècle, les textes théoriques sur la littérature, la musique et le théâtre ont recours aux outils terminologiques rhétoriques ${ }^{8}$.

Il va sans dire que, comme le remarque Georges Décote, les propos argumentatifs de Cazotte concernant la musique se caractérisent par " l'indépendance d'esprit » et que « sa sincérité et sa vigueur sont remarquables »", mais, n'étant pas musicien, mais écrivain, donc n'appartenant pas aux " gens de métier ", Cazotte « donne l'appréciation d'un homme de goût, subjective, mais toujours motivée $»^{10}$. En effet, l'auteur du Diable amoureux ne polémique pas avec les spécialistes en musique, «il ne juge pas en technicien de la musique, il laisse cet aspect aux "gens du métier" " ${ }^{11}$, mais, dans ses propos argumentatifs, se réfère aux arguments rhétoriques. Il place la musique au même niveau que la littérature et les productions dramatiques. Pour lui, ce sont des ouvrages créés avec des règles rhétoriques et qui réalisent les mêmes objectifs que le discours oratoire. Il faut souligner à ce propos que pour les écrivains et les philosophes des Lumières, qu'ils soient ou non auteurs d'ouvrages musicaux, l'opéra est soumis aux mêmes outils critiques que la tragédie ${ }^{12}$, et celle-ci est censée réaliser au moins deux fonctions rhétoriques, le placere et le movere, donc susciter un plaisir esthétique et émouvoir le cœur et l'âme ${ }^{13}$. Il n'est donc pas étonnant que le système terminologique utilisé par Cazotte pour analyser des ouvrages musicaux renvoie directement aux règles de la rhétorique dont se servent les esprits des Lumières pour parler d'autres domaines de l'art.

D'ailleurs, toute la charge d'accusations formulées par Rousseau, qui prennent la forme d'un procès contre la musique française et que Cazotte cherche à réfuter, prennent la forme d'ordre rhétorique. L'auteur de la Lettre sur la musique française soulève le postulat de la finalité de la musique dont le rôle est de par-

7 Encyclopédie ou dictionnaire raisonné des sciences, des arts et des métiers, tome XIV, Neufchastel 1765, p. 250.

8 L'importance et l'universalité de la rhétorique dans les discours scientifiques et critiques au XVIII ${ }^{\mathrm{e}}$ siècle sont mises en valeur par, entre autres, Bernard Lamy, qui explique : « L'Art de parler s'étend (...) à toutes choses. Il est utile aux Philosophes, aux Mathématiciens. La Théologie en a besoin, puisqu'elle ne peut expliquer les vérités spirituelles (...) qu'en les revêtant de paroles sensibles. Certainement nous aurions un plus grand nombre de bons Ecrivains, si on avoit découvert les véritables fondemens de cet Art », B. Lamy, La Rhétorique ou l'art de parler, Paris 1757, p. XIV. Le sujet a été largement débattu dans mon livre La Rhétorique dans la théorie de l'art à l'époque des Lumières en France, Łódź 2014.

9 G. Décote, L'itinéraire de Jacques Cazotte. De la fiction littéraire au mysticisme politique, Genève 1984, p. 66.

10 Ibid.

11 Ibid.

12 L'abbé Batteux ne qualifie pas l'opéra comme un troisième genre de drame, mais fait une division de la tragédie en deux types : la tragédie héroïque et la tragédie merveilleuse qu'est l'opéra et qui, plus qu'aucun autre genre, réalise le movere rhétorique. Les actions, le langage et la musique de l`opéra se caractérisent par des émotions fortes qu'ils transmettent aux spectateurs, Ch. Batteux, Les beaux arts réduits à un même principe, Paris 1746, p. 211-212.

13 Dans son Essai sur l'origine des langues, Jean-Jacques Rousseau énumère trois domaines de la production intellectuelle de l'homme, capable d'agir avec force sur la sensibilité et l'esprit humains. Ce sont l'éloquence, la poésie et la musique, J.-J. Rousseau, Essai sur l'origine des langues, [dans :] Euvres complètes, t. v, textes réunis et présentés par B. Gagnebin et M. Raymond., Paris 1995, p. 411. 
ler et communiquer quelque chose. Puisque c'est à l'âme qu'elle s'adresse, elle peut avant tout communiquer la passion, et elle le fait «par la voix accentuée et infléchie, c'est-à-dire par le chant $\rangle^{14}$. D'après Rousseau, le message communiqué par la musique est renforcé par la langue, qui, dans le cas de la langue italienne, est susceptible d'exprimer les émotions.

Cazotte aborde le problème de la langue dans le contexte de son union avec la musique qui créent ensemble une structure artistique capable d'émouvoir les cœurs. Pour rester impartial, il avoue qu'avant Lulli, les Italiens jouaient un rôle moteur dans le chant et que la musique italienne a servi de modèle aux Français. Pourtant, la France a su retrouver de vraies valeurs dans sa musique, supérieure par rapport à l'italienne parce qu'elle contient « une galanterie » qui est un trait national français et qui réalise non seulement la fonction movere, mais aussi, étant une source de plaisir esthétique, la fonction placere :

Avant Lully, les seuls Italiens chantoient en Europe. L'Italie étoit alors le magasin des Arts, \& principalement de la Musique. Nous y puisâmes comme le reste de l'Europe ; mais nous nous apperçûmes bien-tôt qu'il y avoit dans notre Langue une noblesse, une tournure de sentiment propre à notre caractère particulier, dont la peinture nous feroit plaisir, \& que la modulation Italienne ne rendoit pas ; \& c'est-là l'origine de notre schisme ${ }^{15}$. Peu-à-peu nos Compositeurs en sont venus au point de nous toucher par l'expression fidele des nuances de nos passions ; ils ont rendu jusqu'à cette fleur de galanterie, dont notre nation a été lontems en droit de se piquer. Ils ont trouvé le chemin du cœur, nous ont émû ; ils nous plaisent ${ }^{16}$. Serions-nous sages de négliger les plaisirs réels qui nous viennent de leurs travaux, pour chercher ceux que nous procureroit la Musique Italienne, qui seroient sûrement toujours équivoques pour nous ? ${ }^{17}$

Ce bref aperçu historique de la question ne cherche pas à prouver que la musique française, en s'inspirant de la musique italienne, a progressé pour devenir plus parfaite. Pour Cazotte, la notion de progrès ne se réfère pas à la musique ni à d'autres domaines de l'art. Le progrès et l'évolution sont réservés aux sciences, alors que l'art dépend du génie et de l'imagination créatrice ${ }^{18}$. Pourtant, cela ne signifie pas que la musique n'est soumise à aucune règle. En effet, dans l'argumentation cazotienne, la musique obéit à plusieurs règles pour réaliser des objec-

14 C. Kintzler, Jean-Philippe Rameau. Splendeur et naufrage de l'esthétique du plaisir à l'âge classique, Paris 1983, p. 146.

15 Rousseau porte une opinion tout à fait contraire quant à la modulation française.

16 On observe à ce propos l'influence des postulats de Dubos concernant l'autonomie du public dans l'évaluation de l'œuvre d'art. Dans la rhétorique traditionnelle, qui était encore en vigueur au $\mathrm{XVII}^{\mathrm{e}}$ siècle, la fonction movere vient après la fonction placere. Autrement dit, sans éveiller le plaisir esthétique l'art n'est pas capable d'émouvoir. Cazotte parle d'abord de la musique qui émeut et qui trouve le chemin du cœur; ce n'est qu'après que vient le plaisir.

17 J. Cazotte, op. cit., p. 23.

18 B. Fontenelle, Digression sur les Anciens et les Modernes : Entretiens sur la pluralité des mondes. Digression sur les Anciens et les Modernes, textes réunis et présentés par R. Shackleton, Oxford 1955, p. 166-174. Dans son étude sur l'idée de plaisir esthétique, Agnès Lontrade renchérit sur cette idée : « Le génie se donnant à lui-même ses propres règles et échappant à tout apprentissage par imitation, c'est-à-dire en définitive à toute école comme à tout maître, la notion de progrès en art devient problématique », A. Lontrade, op. cit., p. 140. 
tifs esthétiques et rhétoriques ${ }^{19}$. Bien que Cazotte se range plutôt dans le clan des conservateurs et défenseurs des classiques, qu'il se méfie du parti des Lumières et qu'il n'aime pas Rousseau en repoussant ses arguments, il ne s'obstine pas à défendre aveuglément et à tout prix la musique française, mais, dans la guerre dont il est témoin, il occupe une position analytique pour formuler ses observations.

\section{L'harmonie comme moyen de plaire et d'émouvoir}

Cazotte oppose les notions rhétoriques de plaisir et d'émotion à la primauté de la mélodie sur l'harmonie et des émotions pures et naturelles sur les règles traditionnelles de l'analyse de l'œuvre d'art postulées par Jean-Jacques Rousseau. Défenseur de la tradition en musique, Cazotte est d'avis qu'il n'y a rien de mal à ce que la musique réalise les fonctions rhétoriques en procurant au spectateur/ destinataire du plaisir et de l'émotion.

$\mathrm{Si}$, d'après Rousseau le plaisir venant de la musique doit son origine à la mélo$\operatorname{die}^{20}$, Cazotte voit la chose d'une autre manière. À son avis, c'est grâce à l'harmonie qu'on arrive à produire une composition qui plaît au public. En tant que partie constitutive de l'élocution, l'harmonie se définit « comme un accord de sons qui se suivent $\&$ se lient entr'eux : on peut la comparer au bruit d'un fleuve qui roule ses flots continûment $\&$ sans interruption $»^{21}$.

Cazotte fait une référence à l'harmonie en analysant Titon et l'Aurore de Jean-Joseph de Mondonville, une pastorale-héroïque dont la première se tient au cœur de la polémique engendrée par La serva padrona. Bien que Cazotte soit persuadé de la victoire de l'opéra français sur l'opéra italien (« L’Opera François triomphe »), il se montre impartial et analyse l'ouvrage de Mondonville en prenant en considération plusieurs aspects. En effet, c'est précisément le manque d'harmonie dans l'ouverture qui est son point faible : «L'ouverture est foible. Les connoisseurs la trouvent aussi pleine de fracas qui vuide d'harmonie $»^{22}$. Grâce

19 Cette attitude, d'ailleurs, exprime l'esprit et le goût de l'époque. Par exemple, en parlant des écrivains, Charles Batteux souligne que çest grâce aux règles que l〉ouvrage peut plaire et séduire 1)âme : " Ce sont elles-mêmes [les règles] qui nous apprennent à concilier les sons, à les joindre entr'eux d'une maniere intime ; qui nous montrent les moyens de soutenir l'attention de l'auditeur, de le soulager, de le séduire; en un mot, ce sont elles qui ouvrent l'ame à la persuasion, \& qui font une grande partie de la différence qu'il y a entre les bons \& les médiocres écrivains », Ch. Batteux, De la construction oratoire, Paris 1763, p. 222.

20 C. Kintzler, op. cit., p. 146.

${ }^{21}$ Ch. Batteux, De la construction oratoire..., p. 228. D'après une autre définition de Batteux, la mélodie fait partie de l'harmonie, étant égale au chant : "Toute harmonie est un concert, ou de plusieurs choses qui se suivent avec un certain rapport de liaison, comme quand on fait chanter une seule voix, ou de plusieurs choses qui sont en même tems \& qui concertent ensemble, comme quand deux voix chantent, l'une la basse, l'autre le dessus d'un même air. La première espèce d'harmonie se nomme chant, ou mélodie dans la musique : la seconde espèce retient le nom du genre, \& s'appelle harmonie simplement », Ibid., p. 235-236.

22 J. Cazotte, op. cit., p. 12-13. 
à sa démarche argumentative, Cazotte prévient toute accusation de parti pris qu'on pourrait lui adresser, il est « à la fois engagé et objectif, partisan et impartial $»^{23}$.

Dans un autre fragment, Cazotte met en évidence qu'un arrangement harmonieux des éléments constitutifs de la pièce dans le premier acte du ballet contribue à la création de l'ekphrasis. Cazotte avoue que la force créatrice de la musique l'avait séduit par son pouvoir de peindre des images dans l'imagination du destinataire. Puisque, grâce à la disposition convenable des sons, " rien ne nous étonne », le ballet dans l'ouvrage de Mondonville est « le morceau le plus fleuri, le plus voluptueux que j'aie oui à l'Opéra $»^{24}$. La scène de ballet noue un lien affectif entre l'imagination créatrice de l'artiste et l'imagination représentative du destinataire qui lui fait mettre sous les yeux les images voire toutes les scènes préparées par l'auteur. Elle permet au destinataire de cette œuvre de toucher du doigt la réalité créée par le compositeur et le librettiste :

On croit en même tems voir épanouir les fleurs, tomber la rosée, entendre les gazouillement des oiseaux, sentir le tressaillement de la nature aux approches du jour. La scene qui suit est écrite avec des graces \& du naturel ; elle est terminée par un duo qui ne laisse rien à desirer, \& suivie d'une fête de pastres semée de petits airs tendres \& légers, qui font autant de miniatures ${ }^{25}$.

Chose intéressante, dans sa Lettre sur la musique française, Rousseau explique que c'est la mélodie, grâce à la précision de la mesure, qui rend le chant animé et laisse obtenir une combinaison de sons qui fait voir au spectateur des images, des tableaux ${ }^{26}$. Le même effet est donc réalisé par les deux nations de différentes manières : la mélodie italienne et l'harmonie française.

Il ne faut pas non plus sous-estimer qu'une autre scène dans le même acte, grâce à l'harmonie qui arrange les « bruits ", constitue un exemple de captatio benevolentiae. Puisque les sons arrivent au spectateur d'une manière harmonieuse, il est possible en quelque sorte de séduire son attention : "Une scene de même nature, \& un peu ressemblante, commence à jetter de la froideur dans le second acte. Un chœur de vents plein de bruit \& d'harmonie vient maîtriser l'attention du Spectateur $\gg{ }^{27}$.

Dans le contexte de ses réflexions sur l'harmonie, Cazotte revient au sujet de la relation entre la langue et la musique. En effet, l'air italien, même " isolé de paroles », est agréable en lui-même, et, comme l'avoue Cazotte, l'étranger peut lui donner la préférence. Le problème du chant français dans le contexte de ses rapports avec la langue, est beaucoup plus complexe. La musique française privée

23 G. Décote, op. cit., p. 66.

24 J. Cazotte, op. cit., p. 13.

25 Ibid., p. 12-13.

26 « [L'] avantage (...) qui prête à la mélodie son plus grand effet, est l'extrême précision de mesure qui s'y fait sentir dans les mouvemens les plus lents, ainsi que dans les plus gais : précision qui rend le chant animé et intéressant, les accompagnemens vifs et cadencés, qui multiplie réellement les chants, en faisant d'une même combinaison de sons, autant de différentes mélodies qu'il y a de manieres de les scander; qui porte au cœur tous les sentimens, et à l'esprit tous les tableaux ; qui donne au Musicien le moyen de mettre en air tous les caractères de paroles imaginables, plusieurs dont nous n'avons pas même l'idée », J.-J. Rousseau, Lettre sur la musique française..., p. 303-304.

27 J. Cazotte, op. cit., p. 15. 
de paroles est une sorte d'unité harmonieuse, ce qui d'un côté, peut plaire aux connaisseurs dont les oreilles et surtout les esprits sont habitués à la réception d'un « trait d'harmonie privé de sa base fondamentale », mais, de l'autre côté, risque de constituer un défaut dans le cas du destinataire non préparé théoriquement.

Pour rester dans l'atmosphère de guerre, Cazotte énumère les victimes de ce conflit acharné. Il y a des morts des deux côtés (par exemple Acis et Galathée du côté des Français et La finta Cameriera du côté des Italiens). Mais, ce qui est intéressant du point de vue de l'argumentation se référant à l'harmonie, Titon et Aurore est considéré comme une victime qui a été blessée en recevant deux coups, dont le premier a été dû, effectivement, à quelques imperfections concernant l'harmonie :

«Le Ballet de Titon a reçu deux profondes blessures, l'une dans son corps d'harmonie, l'autre dans la partie de l'invention. Le goût a un peu souffert de la démarche qu'il a faite, en voulant concilier les deux Musiques $»^{28}$.

Malgré cette " blessure », l'opéra de Mondonville remporte un succès important. Il est repris en 1754, 1763, 1764 et 1768. Il est aussi joué à Bruxelles (1754), à Parme (1758), à Cassel (1767) et à Marseille (1777). Après deux siècles d'éclipse, il revoit le jour en 1991, à Reims et au Palais Garnier, sous la direction de Marc Minkowski.

\section{La séduction esthétique comme critère de la valeur artistique}

Mais l'ouvrage de Mondonville ne doit pas seulement ce succès à sa composition harmonieuse. En répondant au goût de l'époque, Titon et Aurore éveille chez le public des émotions profondes qui, au XVIII ${ }^{\mathrm{e}}$ siècle, constituent un trait distinctif dans la critique de l'art. En effet, selon Dubos, la nature a crée la sensibilité du cœur humain et sa disposition à se laisser émouvoir par l'art de l'imitation. Cette sensibilité est l'arbitre de la qualité de l'ouvrage. La raison peut porter un jugement sur son adaptation aux règles, mais ce ne sont pas ces dernières qui décident de la qualité de l'œuvre ; elle peut être médiocre même si les critiques ne lui reprochent aucun écart par rapport aux principes de création. Dubos souligne que c'est notre sensibilité qui nous renseigne sur la valeur de l'ouvrage. Il pose d'abord en principe que l'art a pour but de plaire et de toucher. Rousseau, qui renchérit sur cette idée, indique que les passions constituent la source des langues primitives. Il affirme : «Avec les prémiéres voix se formérent les prémiéres articulations ou les prémiers sons, selon le genre de la passion qui dictoit les uns ou les autres $»^{29}$.

Cazotte réagit à toute une vague de critiques qui s'étendent en France et qui sont dirigés contre la musique française, incapable, d'après Gluck et trois philosophes dont Cazotte ne révèle pas les noms, d'éveiller des sentiments positifs :

\footnotetext{
28 Ibid., p. 24.

29 J.-J. Rousseau, Essai sur l'origine des langues..., p. 410.
} 
Un Allemand avoit imaginé que nos Danseurs exécutoient de mauvaise grace, que notre Chant ne rendoit point l'expression de notre Langue. Et trois Géometres avoient calculé que le tout, joint ensemble, ne pouvoit pas faire une somme complette de plaisir ${ }^{30}$.

En premier lieu, l'auteur de la Guerre indique l'absence de sensibilité chez les « Géometres » qui leur rend impossible d'apprécier une réelle faculté de la musique française de plaire au public. Extrêmement insensibles, ils sont incapables de réagir avec bienveillance aux productions musicales françaises : «J'ai oui-dire qu'on ne les faisoit éternuer qu'avec de la bétoine. Puisqu'ils sont si durs à émouvoir, les croyez-vous faits pour mesurer nos amusemens ? $»^{31}$.

Ensuite, Cazotte avoue que, certes, la musique italienne peut plaire, mais seulement à ceux qui ignorent les règles de la musique :

Nous n'envions point aux étrangers le plaisir qui résulte pour eux de la violation des regles $\&$ des bienséances ; c'est à elles que nous devons des chefs-d'œuvres dans tous les genres, $\&$ nous nous sommes fait une loi de les respecter même sur le Théatre ${ }^{32}$.

C'est un argument qui consitue la continuation de tout un raisonnement cazotien quant à l'harmonie considérée comme un arrangement convenable des compostantes de l'ouvrage. Cette fois-ci, l'harmonie contribue à la réalisation de la fonction esthétique.

Dans sa discussion sur l'opéra comme un moyen de plaire et d'émouvoir, Cazotte se réfère aux arguments déjà abordés pour, d'un côté, les développer et, de l'autre, les préciser en en atténuant la vivacité peut-être trop perçante. Il s'agit, en effet, des deux «blessures » qu'a reçues Titon et Aurore, la première dans le domaine de l'harmonie et l'autre, en matière de l'invention, donc de la partie de la rhétorique responsable du choix convenable de la matière à traiter et des lieux à explorer. Cazotte s'oppose à l'attitude des philosophes à mépriser les sujets mythologiques dans les opéras français. Certes, avoue-t-il, « Dieux du Paganisme » et « les rites \& les cérémonies des Anciens » y sont altérés, mais, ayant au choix le paganisme dans $\operatorname{Sethos}^{33}$ et celui dans l'opéra, Cazotte préfère ce dernier parce que la fiction dans le spectacle lyrique lui donne la possibilité de se délecter du plaisir esthétique. Dans le cas du spectacle d'opéra, l'élément essentiel, c'est le sujet. Il décide du succès ou de la chute de ce spectacle. D'après un autre défenseur de l'opéra français, Francesco Algarotti, le sujet est comparé à une toile « sur laquelle est dessiné le tableau qui est ensuite colorié par le Musicien, \& fini par les autres $»^{34}$. D'après Algarotti, qui, en quelque sorte, répète l'argumentation de Cazotte, les motifs historiques ne sont pas aussi bons que les thèmes mythologiques parce que « les sujets Historiques sont le plus souvent stériles, froids \& languissans », ce qui s'oppose à l'idée du spectacle d'opéra qui se veut «magnifique \& imposant ${ }^{35}$. De même, comme le remarque Cazotte, les

30 J. Cazotte, op. cit., p. 6.

31 Ibid.

32 Ibid., p. 20.

33 Cazotte se réfère ici au roman de Jean Terrrasson publié en 1731 et étant une imitation de Télémaque. Dans Sethos, la fiction sert à enseigner une morale indépendante de la religion.

34 F. Algarotti, Essai sur l'opéra, Pise-Paris 1773, p. 10.

35 Ibid., p. 18. 
sujets qui s'inspirent de l'antiquité constituent un trait caractéristique de l'opéra et il faut les laisser exister :

Quant aux Dieux du Paganisme, qui n'ont plus d'autels qu'à l'Opéra, je ne vois pas l'intérêt qu'ont les Géometres à les en chasser. Ils ne leur causent point d'embarras ; pourquoi les troubler dans leur petite Juridiction?

D'ailleurs, tout altérés que soient au Spectacle de l'Opéra les rites \& les cérémonies des Anciens, ce qu'on y en voit pique ma curiosité. Je sens bien ce qu'on pourroit faire de plus pour la satisfaire ; mais je me contente de ce que je trouve-là ; \& les images toutes imparfaites qu'elles sont, me plaisent plus que ce que je pourrois lire à ce sujet dans Sethos, ou dans quelque livre encore plus ennuyeux ${ }^{36}$.

Les sujets mythologiques, tant méprisés par certains, dans le cas de l'opéra français, grâce à leur exploitation consciente et créative, permettent donc de fournir au public un vrai plaisir et de l'inciter à aller voir le spectacle, ce qui, dans l'argumentation cazotienne est encore une preuve de la victoire des Français sur les Italiens dans cette guerre.

On ne peut pas non plus faire abstraction de l'importance d'une fonction rhétorique qui, d'après Cazotte, constitue la source d'un profond plaisir esthétique et contribue au fait que « les étrangers perdirent la bataille ». Il s'agit de l'actio, la partie de la rhétorique qui, dans le cas du spectacle, concourt à l'effet scénique, c'est-à-dire le débit, les gestes et les mouvements de l'acteur. En effet, une voix sonore et vibrante, une physionomie vive, des gestes expressifs et appropriés au sujet permettront à l'acteur d'éveiller des émotions vives et sincères chez les spectateurs.

Bien sûr, la voix n'est pas l'unique composante de l'actio. L'acteur ne reste pas immobile, il persuade aussi par les mouvements de son corps. D'après Rousseau, dans le cas de l'opéra, une actio parfaite résulte d'abord d'une union harmonieuse de la musique et de la voix. Si cette harmonie est réussie, elle exerce une influence directe sur les gestes qu'elle détermine :

[La] parfaite correspondance de la symphonie et du chant (...) fait que tous les traits qu'on admire dans l'une, ne sont que des développemens de l'autre, desorte que c'est toujours dans la partie vocale qu'il faut chercher la source de toutes les beautés de l'accompagnement. Cet accompagnement est si bien un avec le chant, et si exactement rélatif aux paroles, qu'il semble souvent déterminer le jeu et dicter à l'Acteur le geste qu'il doit faire ${ }^{37}$.

Cazotte réagit défavorablement à la manière de chanter de l'acteur italien. Tout en avouant que le bel canto a du charme, il lui reproche d'en négliger les principes ce qui, en conséquence, conduit au dégoût : « Sa voix n'est ni forte ni flatteuse, ni naturelle. Il semble ne point connoître le beau goût du Chant Italien, aussi n'est-ce par le droit de sa charge : ce n'est qu'un Bouffon $»^{38}$. Ensuite, il remarque que non seulement l'exécution de l'ouvrage par les chanteurs italiens ne fait pas plaisir, leur chant est dépourvu de variété capable d'intéresser les spectateurs, mais encore elle représente une violation des règles du bon chant. De

36 J. Cazotte, op. cit., p. 8.

37 J.-J. Rousseau, Lettre sur la musique française..., p. 306.

38 J. Cazotte, op. cit., p. 5. 
la comparaison des acteurs italiens et des acteurs français, ces derniers sortent gagnants parce que leur concurrents italiens n'ont aucun charme dont les Français sont naturellement dotés :

La toile se leve, le Spectacle commence ; la farce Italienne étoit misérable pour la conduite, le Chant moins varié que celui des précédens intermedes.

On voit des Acteurs dépourvûs, je ne dis pas de graces Françoises ; on peut n'en avoir pas l'air \& les manieres; mais les belles habitudes du corps sont de tout pays. Du reste rien ne dédommageoit en eux de la mauvaise contenance.

Enfin paroît ce chanteur si vanté, dont la voix fit peu de plaisir, dont l'état causa à la plus intéressante partie de l'assemblée un dégoût mêlé d'indignation, \& fit pitié à tout le reste ${ }^{39}$.

La conclusion de Cazotte, maintes fois reprise, quant au résultat de la guerre, ne laisse pas de doute : " Les étrangers perdirent la bataille » dit-il à propos des représentants du goût italien dans la musique. Cette déclaration peut être considérée comme trop ferme par des gens de métier et des spécialistes en musique. Mais Cazotte n'est pas musicien et sa polémique constitue un exemple de discours argumentatif sur l'art en général, exprimé par un représentant de l'esthétique des Lumières en France, pour qui les règles de la rhétorique servent tout naturellement d'instrument d'analyse de l'œuvre d'art. D'un côté plongé dans la tradition intellectuelle de la France d'avant la Révolution, de l'autre, étant l'expression de la sensibilité émotive de l'époque qui va venir, La Guerre de l'opéra est le résultat d'une volonté, allant presque vers une exigence absolue, de prendre parole au sujet qui engage maints esprits dans la deuxième moitié du XVIII siècle.

\section{Bibliographie}

Algarotti F., Essai sur l'opéra, Pise-Paris 1773.

Batteux Ch., De la construction oratoire, Paris 1763.

Batteux Ch., Les beaux arts réduits à un même principe, Paris 1746.

Dubos J.-B., Réflexions critiques sur la poésie et la peinture, seconde partie, Paris 1719.

Cazotte J., La Guerre de l'opéra. Lettre écrite à une dame de province, [sans lieu] 1753.

Décote G., L’itinéraire de Jacques Cazotte. De la fiction littéraire au mysticisme politique, Genève 1984.

Encyclopédie ou dictionnaire raisonné des sciences, des arts et des métiers, tome XIV, Neufchastel 1765.

Fontenelle B., Digression sur les Anciens et les Modernes : Entretiens sur la pluralité des mondes. Digression sur les Anciens et les Modernes, textes réunis et présentés par R. Shackleton, Oxford 1955.

Kaminski P., Mille et un opéras, Paris 2004.

Kintzler C., Jean-Philippe Rameau. Splendeur et naufrage de l'esthétique du plaisir à l'âge classique, Paris 1983.

Lamy B., La Rhétorique ou l'art de parler, Paris 1757. 
Lontrade A., Le plaisir esthétique. Naissance d'une notion, Paris-Budapest-Torino 2004.

Nuitter Ch., Thoinan E., Les Origines de l'Opéra français, Paris 1886.

Rousseau J.-J., Essai sur l'origine des langues [dans :] Euvres complètes, t. V, textes réunis et présentés par B. Gagnebin et M. Raymond, Paris 1995.

Rousseau J.-J., Lettre sur la musique française [dans :] Euvres complètes, t. V, textes réunis et présentés par B. Gagnebin et M. Raymond, Paris 1995.

Zacharow S., La Rhétorique dans la théorie de l'art à l'époque des Lumières en France, Łódź 2014. 